\title{
Chronic Obstructive Pulmonary Disease and Obstructive Sleep Apnea-Overlap Syndrome and its Cognitive Impairments
}

\author{
Johnson $\mathrm{S}^{1 *}$ and Alex J² \\ ${ }^{1}$ Department of Respiratory Therapy, Inaya Medical College, Riyadh, Kingdom of Saudi Arabia \\ ${ }^{2}$ Clinical Psychology, Department of Behavioral Sciences, College of Medicine, Dar Al Uloom University, Riyadh, Kingdom of Saudi Arabia \\ *Corresponding author: Johnson S, Assistant Professor and Vice Chair, Department of Respiratory Therapy, Inaya Medical College, Riyadh, Kingdom \\ of Saudi Arabia Tel: 966-556387268; E-mail: sjohnson@inaya.edu.sa
}

Received date: March 06, 2018; Accepted date: March 07, 2018; Published date: March 14, 2018

Citation: Johnson S, Alex J (2018) Chronic Obstructive Pulmonary Disease and Obstructive Sleep Apnea-Overlap Syndrome and its Cognitive Impairments. Chron Obstruct Pulmon Dis Vol No. 3 Iss No. 1:31.

Copyright: (C) 2018 Johnson S et al. This is an open-access article distributed under the terms of the Creative Commons Attribution License, which permits unrestricted use, distribution, and reproduction in any medium, provided the original author and source are credited.

\section{Editorial}

Chronic obstructive pulmonary disease is a very prevalent disorder in the western world and also in the developing countries. It accounts for a prevalence of $13.9 \%$ in the adult population in USA and it is the fourth leading cause of death in USA [1,2]. Chronic obstructive pulmonary disease (COPD) and obstructive sleep apnea (OSA) are extremely prevalent conditions; this makes the chance of overlap syndrome. Obstructive sleep apnea is a sleep disorder that comprises of cessation or substantial decrease in airflow in the presence of breathing effort. It is the most common type of sleep-disordered breathing and is characterized by repeated episodes of upper airway collapse during sleep [3]. These episodes are accompanied with repeated oxyhemoglobin desaturations and arousals from sleep. The prevalence of obstructive sleep apnea (OSA) seems to be $9 \%$ to $26 \%$ in adult population and is diagnosed by polysomnography. An Apnea Hypopnea Index (AHI) $<5 /$ hour is considered normal, AHI $5-10 /$ hour is mild, $10-30$ /hour is moderate and $>30 /$ hour as severe.

Patients who have overlap syndrome of chronic obstructive pulmonary disease (COPD) and obstructive sleep apnea (OSA) have worse prognosis if compared to the patients who may have just one of the diseases [4] and greater risk of prolonged hypoxemia during night when compared with OSA but without COPD [5]. Marin and coworkers reported that OSA increases mortality rate in patients with COPD also in patients who are suspected to have overlap syndrome does not matter if they are on CPAP therapy or not [6].

There are factors that can complicate the disease in overlap syndrome. They are in (COPD patients) diminished sleep quality, hypoxemia, obstructive sleep apnea (OSA) the rostral fluid shift from supine, cigarette smoking, medications such as corticosteroids. There are some factors that are protective against OSA, they are Low BMI, reduced REM sleep and Theophylline [7].

The relationship between COPD and cognitive impairment is a well-known aspect. The low peripheral oxygen saturation ( $\leq 88 \%)$ and hypoxia is associated with the risk of cognitive impairment in COPD patients. The neuropsychological tests are related to the level of cortical and subcortical level of function. The tests are primarily assessing executive functions, language functions, social cognitions and emotions, attention and concentration, learning and memory, visuospatial motor functions. The scientific studies explicitly show significant deficits in the area of memory and attention in neuropsychological tests. Especially verbal memory and learning area has predominantly affected and impaired. Similarly, visuospatial and intermediate memory also demonstrates substantial impairment. According to the severity of COPD, the cognitive dysfunction may change and it will affect the quality of life of the individual. In this context, Neuropsychological evaluation should be made as a part of routine evaluation of COPD patients and thus we can identify cognitive deficits early and prevent further cognitive decline. Mini-Mental State Examination (MMSE) [8], Wechsler Memory Scale-III, Wechsler Adult Intelligence Scale-III [9], Rey Auditory Verbal Learning Test (RAVLT) [8] are the common cognitive function tests employed in COPD patients.

Obstructive sleep apnea (OSA) is associated with cognitive impairment. The chief factors of cognitive deficits in OSA are sleep disruption and blood gas abnormalities. COPD and OSA have deficits in attention, memory, executive function, psychomotor function, language abilities, suggestive of hypoxia/ hypercarbia being important determinant of deficits in these domains in OSA [10].

\section{References}

1. Jemal A, Ward E, Hao Y, Thun M (2005) Trends in the leading causes of death in the United States, 1970-2002. JAMA 294: 1255-1259.

2. Mannino DM, Gagnon RC, Petty TL, Lydick E (2000) Obstructive lung disease and low lung function in adults in the United States: data from the National Health and Nutrition Examination Survey, 1988-1994. Arch Intern Med 160: 1683-1689.

3. Young T, Palta M, Dempsey J, Skatrud J, Weber S, et al. (1993) The occurrence of sleep-disordered breathing among middle-aged adults. N Engl J Med 323: 1230-1235. 
4. Chaouat $A$, Weitzenblum $E$, Krieger J, Ifoundza $T$, Oswald $M$, et al. (1995) Association of chronic obstructive pulmonary disease and sleep apnea syndrome. Am Rev Respir Dis 151: 82-86.

5. Sanders $M H$, Newman $A B$, Haggerty $C L$, Redline $S$, Lebowitz $M$, et al. (2003) Sleep and sleep-disordered breathing in adults with predominantly mild obstructive airway disease. Am J Respir Crit Care Med. 167: 7-14.

6. Marin JM, Soriano JB, Carrizo SJ, Boldova A, Celli BR (2010) Outcomes in patients with chronic obstructive pulmonary disease and obstructive sleep apnea: the overlap syndrome. Am J Respir Crit Care Med 182: 325-331.

7. McNichola WT (2016) Chronic obstructive pulmonary disease and obstructive sleep apnoea-the overlap syndrome. J Thorac Dis 8: 236-242.
8. Villeneuve S, Pepin V, Rahayel S, Bertrand JA, de Lorimier M, et al. (2012) Mild cognitive impairment in moderate to severe COPD: A preliminary study. Chest 142: 1516-1523.

9. Dodd JW, Charlton RA, van den Broek MD, Jones PW (2013) Cognitive dysfunction in patients hospitalized with acute exacerbation of COPD. Chest 144: 119-127.

10. Olaithe M, Bucks RS, Hillman DR, Eastwood PR (2018) Cognitive deficits in obstructive sleep apnea: Insights from a meta-review and comparison with deficits observed in COPD, insomnia, and sleep deprivation. Sleep Med Rev 38: 39 - 49. 